\title{
UNIQUE CHANGES IN THE RETINAL VEINS OF A DIABETIC PATIENT*
}

\author{
BY
}

\author{
M. H. LUNTZ AND JOAN HICKLEY
}

Nuffield Laboratory of Ophthalmology, Oxford, and Cape Town, Union of South Africa

THIS paper illustrates three forms of loop formation in the retinal veins of a diabetic patient, two of which have not to the best of our knowledge previously been described.

A prominent feature of diabetic retinopathy is early engorgement and congestion of the retinal veins. Tortuosity of these vessels suggests that they are also elongated. Localized venous engorgement, called "beading", is a common manifestation. That this engorgement and congestion are associated with venous stasis is suggested by the presence of new vessel formation and haemorrhage which may organize and form areas of retinal proliferation. In some instances this proliferation is pre-retinal, occurring without any preceding haemorrhage. This type can be readily distinguished from that following a vitreous haemorrhage such as occurs in retinal periphlebitis. In the former, fine connective tissue webs filled with delicate newly-formed vessels invade the vitreous, usually from the disc; in the latter, fibrous membranes showing only scanty vessels form bands in front of the retina. These are generally connected to the disc and are classically described as "invading" the vitreous. The observations of Hruby (1954) suggest, however, that this is not so; he postulates that the vitreous body in these cases retracts and that the fibrous bands stretch from the retina to the posterior hyaloid membrane in the retro-vitreal space. Ballantyne and Loewenstein (1943) emphasized that the major changes in diabetic retinopathy occurred in the retinal veins (and our retinal photographs suggest that this is so), and illustrated the changes seen in patients over the previous 7 years in the Western Infirmary and Tennant Institute, Glasgow. Prominent amongst these changes were distension and "beading" of the veins, which were still found in the absence of retinitis and other arterial changes (Ballantyne, 1939). These authors also described small unclosed loops in the veins and coils formed by branching vessels. These loops are well illustrated and form the only well-described series we were able to find in the literature. Some occurred in areas of retinitis proliferans and were probably the result of traction on the vein, but some occurred independently of this,

That similar loops may be secondary to retinitis proliferans as such and independent of a diabetic retinopathy has recently been shown by Davanger (1961), who described pre-retinal loops in retinal veins in a case of retinitis 
proliferans which he ascribed to a tuberculous aetiology and which followed a vitreous haemorrhage. Davanger postulates that the haemorrhage was followed by a partial detachment of the vitreous body, as described by Hruby (1954), the vitreous remaining attached at a few points, presumably the sites of the periphlebitic foci. Contraction of fibrous bands between the retina at these sites and the posterior hyaloid membrane raises the retinal veins into pre-retinal loops. The veins are dissected free along the potential cleft between Krückman's limitans perivascularis and the adventitia of the vessel wall, so that the retina does not detach.

Ballantyne and Loewenstein (1943) and Davanger (1961) described loops which are similar in appearance. Recently we have seen a diabetic patient whose fundi showed loops similar to those already described as well as others not previously described. We felt that it would be of value to report these, and we suggest that they are not isolated pathological phenomena but represent grades of severity in a single disease process.

The abnormalities were photographed with a Zeiss retinal camera, the first and second being from the right fundus, and the third from the left fundus. In the right fundus the loops are not related to any areas of retinitis proliferans, so that they cannot result from fibrous tissue contraction, as in Davanger's case.

\section{Case Report}

A 63-year-old white male was first noted to have diabetes in 1945, when his fasting blood sugar was $132 \mathrm{mg}$. per cent. Diabetic retinopathy was first found in August, 1957, and during the intervening period his diabetes had at times been uncontrolled; he had also passed "gravel" in his urine and renal calculi were found. In 1957 his fasting blood sugar was $209 \mathrm{mg}$. per cent. and he was put on to a regime of diet and Tolbutamide.

He was first seen by us in February, 1961, when the diabetes was under control, the blood pressure was 174/90, and he had a hepato-splenomegaly for which he refused further investigation.

Ophthalmological Examination.-The visual acuity was 6/9 in the right eye with correction, and hand movements in the temporal field only in the left eye. The fundi showed a typical advanced diabetic retinopathy with a few areas of retinitis proliferans in the right eye and widespread proliferans and macular scarring in the left eye. Venous congestion was prominent, as were the changes which form the subject of this paper (Figs 1-3, opposite).

Fig. 1 shows a small crescent-shaped loop in a retinal vein, with the ends of the crescent almost meeting. There is no retinitis proliferans in the region where the loop has formed and it lies in the retina. This picture is similar to that described by Ballantyne and Loewenstein (1943).

Fig. 2 shows a loop (again in an area where there is no retinitis proliferans) which is completely closed and appears to lie in front of the vein as though it had twisted around its base at its point of origin from the vein.

Fig. 3 illustrates two apparently isolated vascular loops in an area of retinochoroidal scarring. Careful examination of the retina with a Hruby lens and slit lamp showed fine capillary vessels running from the vessels adjacent to these loops and presumably maintaining a circulation with them. These capillaries are not visible in the illustration. 


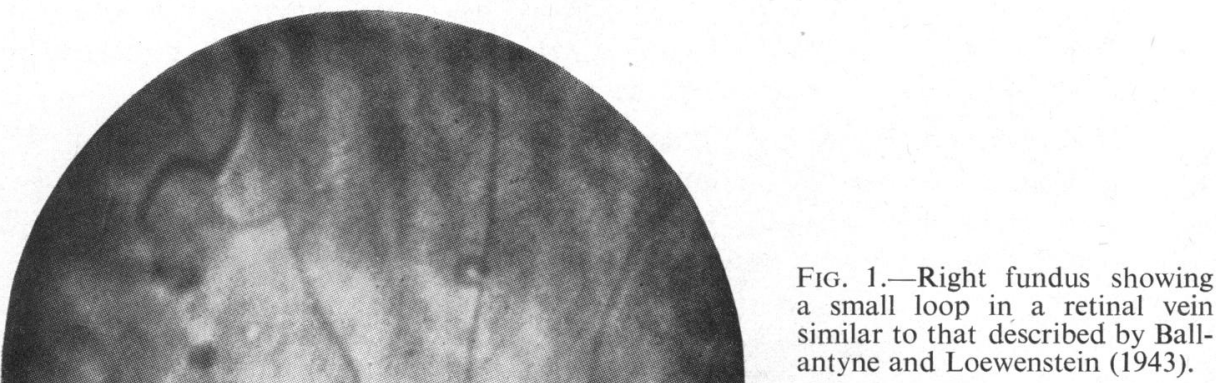

Fig. 2.-Right fundus, showing loop in retinal vein completely closed. It appears to lie in front of the vein as though it had twisted around its base at its point of origin. antyne and Loewenstein (1943).

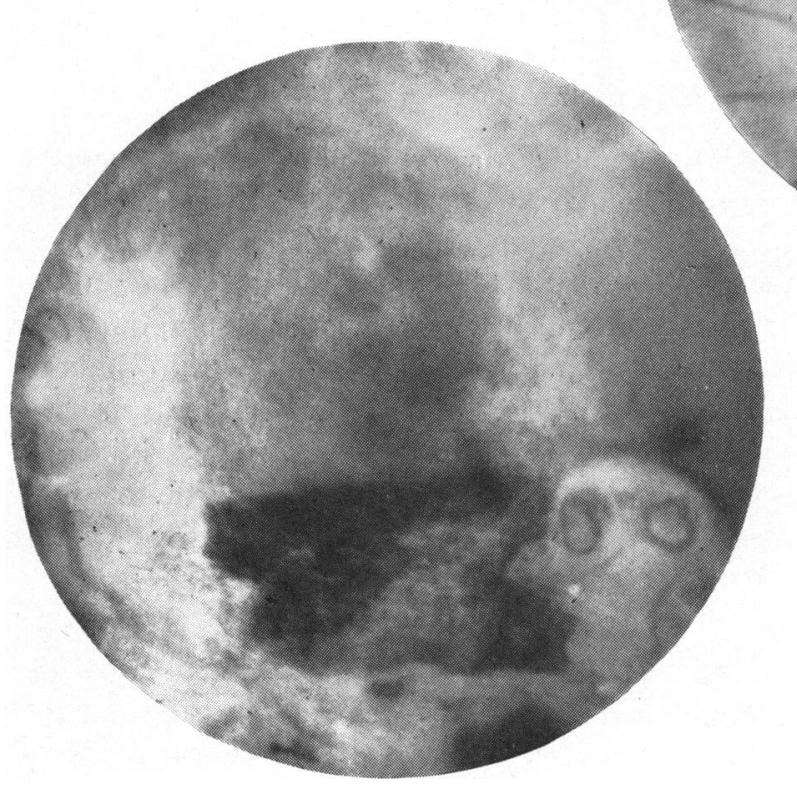

FIG. 3.- Left fundus, showing two apparently isolated loops in an area of retino-choroidal scarring. 
Figs 1 and 2 thus demonstrate loops of the retinal veins occurring independently of retinitis proliferans, and Fig. 3 is interesting as it demonstrates what appear to be isolated vascular loops, connected to the veins by fine capillaries.

A retinal photograph (Fig. 4), taken on January 1, 1962, after an interval of 7 months, shows a considerable increase in the diabetic retinopathy. The photograph shows the same area as that seen in Fig. 1, and the loop of retinal vein clearly seen in Fig. 1 is now almost obliterated by organized fibrous tissue.

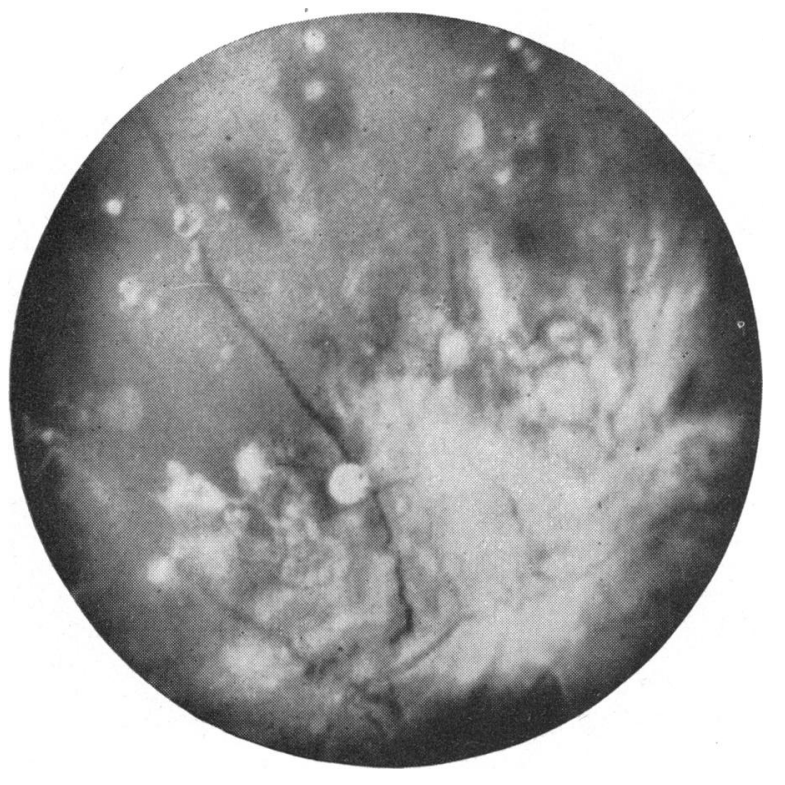

FiG. 4.-Right fundus, showing increased diabetic retinopathy after an interval of 7 months.

\section{Discussion}

Whether these are isolated abnormalities or have some inter-connection one does not know. It is tempting to consider them as different grades of the same pathological process. There is, of course, no evidence for this apart from the fact that they all occurred in the fundus of the same patient and would thus appear to be related. A suggested sequence for linking these three abnormalities is that in certain circumstances where a vein in diabetic retinopathy becomes elongated and congested in a localized area, as they often do, a loop forms at this site. This implies that the vein is partly fixed so that it forms the loop instead of following a tortuous course. The maximal circulatory congestion will occur at the distal end of the loop which widens so that the open proximal mouth becomes narrower (Fig. 1).

Elongation and congestion of the affected portion will then continue so that the loop becomes larger and its mouth narrower. Engorgement of the loop will be limited by the inelastic adventitia of the vessel wall so that a point is reached where it is forced to twist about its base, and the loop is forced 
forwards in front of the vein (Fig. 2). Dissection of the cleft between Krückman's limitans perivascularis and the adventitia, as Davanger (1961) suggests, will prevent the retina from detaching. If the loop twists slowly enough, the base will gradually strangulate and slough, and during this time new vessels will grow into the increasingly anoxic loop from the adjacent vein. A later stage is seen in Fig. 3, where the vascular loops appear to be isolated but are in fact connected by fine capillaries to the adjacent vessels.

\section{Summary}

Three forms of loop formation in the retinal veins of a diabetic patient are illustrated and described. The changes shown in Figs 2 and 3 have not previously been noted in the literature of diabetic retinopathy.

It is suggested that these are not unconnected phenomena, but represent grades of a single pathological process.

Our thanks are due to Dr. A. Landau, Cape Town, who supplied details of the patient's general condition and the treatment he was receiving.

\section{REFERENCES}

Ballantyne, A. J. (1939). Trans. ophthal. Soc. U.K., 59, 226. and LoEWENSTEIN, A. (1943). Ibid., 63, 95.

Davanger, M. (1961). Acta ophthal. (Kbh.), 39, 1. HRUBY, K. (1954). Proc. roy. Soc. Med., 47, 163. 